\title{
Determinación de esteroles y dialcoholes triterpénicos en aceite de oliva mediante separación de la fracción por cromatografía líquida de alta eficacia y análisis por cromatografía de gases. Estandarización del método analítico
}

\author{
Por Arturo Cert (1), Wenceslao Moreda (1) y Josep García-Moreno (2) \\ (1) Instituto de la Grasa (C.S.I.C.). Avda. Padre García Tejero 4. Sevilla. E-41012. España \\ (2) Agra, S.A., Avda. Pius XI, 24. Gandía (Valencia). E-46700. España
}

RESUMEN

Determinación de esteroles y dialcoholes triterpénicos en aceite de oliva mediante separación de la fracción por cromatografía líquida de alta eficacia y análisis por cromatografía de gases. Estandarización del método analítico.

Se presenta un método para la determinación conjunta de esteroles y dialcoholes triterpénicos (eritrodiol y uvaol) en aceites de oliva que consta de las etapas siguientes: obtención del insaponificable, fraccionamiento mediante cromatografía líquida de alta eficacia (CLAE) en régimen isocrático con detector de índice de refracción, preparación de derivados sililados y análisis por cromatografía de gases en columna capilar. Se comparan cuatro procedimientos que utilizan el análisis por cromatografía de gases de las fracciones de esteroles y de esteroles + dialcoholes obtenidas tanto por CLAE como por CCF. Se lleva a cabo un análisis colaborativo para la determinación de esteroles comparando los métodos CLAE-esteroles+dialcoholes y CCFesteroles (método oficial). Los resultados mostraron que por el primer procedimiento la determinación de $\Delta 7$-esteroles se realiza con mayor repetibilidad y reproducibilidad, aunque los valores resultantes son más altos. No se encontraron diferencias en los parámetros de precisión ni en los valores de los restantes esteroles. Las determinaciones de eritrodiol+uvaol por los métodos CLAE-esteroles+dialcoholes y CCF-esteroles +dialcoholes (método oficial) resultaron con similar precisión.

PALABRAS-CLAVE: Aceite de oliva - Esteroles - Eritrodiol Estudio colaborativo - Uvaol.

\section{SUMMARY}

Determination of sterols and triterpenic dialcohols in olive oils using HPLC separation and GC analysis. Standardization of the analytical method.

A method is developed for the analysis of sterols together with triterpenic alcohols (erythrodiol and uvaol) in vegetable oils. The method involves obtention of the unsaponifiable matter, isocratic HPLC separation with refractive index detector, preparation of silylderivatives, and analysis by capillary GC. Four procedures involving $\mathrm{GC}$ analysis of sterol and sterol+dialcohol fractions obtained by HPLC and CCF separations are compared. A collaborative trial comparing HPLC-sterols+dialcohols and CCF-sterols (official) methods is carried out. For $\Delta 7$-sterols, higher values and better repeatability and reproducibility were observed in the first method, but no differences for the remainder sterols were found. The erythrodiol+uvaol determinations by HPLC-sterols+dialcohols and CCF-sterols+dialcohols (official) methods resulted in similar precision.
KEY-WORDS: Collaborative trial - Erythrodiol - Olive oil Sterols - Uvaol.

\section{INTRODUCCIÓN}

La composición de la fracción esterólica del aceite de oliva es un parámetro de gran utilidad para detectar adulteraciones por la presencia de otras grasas y aceites. Así, mediante la determinación de colesterol, brasicasterol, campesterol, estigmasterol, $\beta$-sitosterol y $\Delta 7$-estigmastenol puede detectarse la adición de las grasas siguientes: una proporción muy baja (aproximadamente el $1 \%$ ) de grasa animal y de los aceites de cártamo, colza, germen de trigo, girasol, maíz y sésamo; una cantidad algo mayor (2-3\%) de los aceites de jojoba, lino, soja y semilla de té; porcentajes mayores $(5-9 \%)$ de manteca de cacao y de aceites de algodón, cacahuete, palma y pepita de uva; $y$ alrededor del $20 \%$ de aceite de avellana. Asimismo, la suma de los alcoholes triterpénicos eritrodiol y uvaol es de utilidad para detectar la presencia de aceite de orujo. En consecuencia los reglamentos sobre el aceite de oliva de la Comunidad Europea y del Consejo Oleícola Internacional, y el Codex Alimentarius de la FAO/OMS establecen límites para los porcentajes de estos compuestos.

La determinación de esteroles y eritrodiol+uvaol actualmente se realiza mediante dos métodos similares que constan de las siguientes etapas: a) obtención y purificación del insaponificable; b) separación de las fracciones de esteroles y de alcoholes triterpénicos mediante cromatografía en capa fina de sílica gel (CCF); c) raspado de las bandas correspondientes y extracción de los compuestos adsorbidos sobre la sílica gel: d) preparación de los derivados silanizados; y e) análisis por cromatografía de gases en columna capilar sobre fase de 5\%-fenilmetilsilicona o similar. Para la determinación de esteroles se raspa únicamente la banda que contiene estos compuestos mientras que para la determinación de eritrodiol+uvaol se raspan conjuntamente las bandas de esteroles y dialcoholes triterpénicos; por tanto hay que repetir la etapa de separación en CCF y siguientes, con el consiguiente costo de tiempo y dinero. 
Por otra parte, tal cantidad de operaciones da lugar a que la determinación de los esteroles que se encuentran en baja proporción (colesterol, brasicasterol y $\Delta 7$-estigmastenol) esté afectada de falta de repetibilidad y, sobre todo, de reproducibilidad (Morchio et al., 1989; Moreda et al., 1995). Especialmente delicado es el caso del $\Delta 7$ estigmastenol que se encuentra en los aceites de oliva en proporciones $(0,2-0,4 \%)$ cercanas al límite máximo permitido $(0,5 \%)$ y cuya determinación resultó con una desviación estándar relativa de la repetibilidad $\left(R S D_{r}\right)$ entre 13 y $24 \%$ y una desviación estándar relativa de la reproducibilidad $\left(R S D_{R}\right)$ entre 26 y $36 \%$. León y Cert (1994) en un estudio sobre el método analítico encuentran que la insuficiente separación en CCF entre la banda de esteroles y alcoholes triterpénicos, la delimitación de la banda de esteroles en la CCF y las impurezas cercanas a la banda de los dialcoholes triterpénicos son las principales causas de esta falta de precisión.

Recientemente, Biedermann et al. (1993) han puesto a punto un método para la determinación de esteroles en el que se transesterifica el aceite mediante metilato sódico, la mezcla de reacción se extrae con hexano y el extracto se inyecta en un sistema de cromatografía líquida de alta eficacia acoplado con un cromatógrafo de gases (CLAE-CG). Este procedimiento junto con el oficial fue aplicado a 30 muestras (Lanuzza et al., 1995) obteniéndose resultados similares, aunque no se realizó estudio estadístico para analizar la significación de las diferencias. Este método tiene dos claras ventajas, la eliminación de la etapa de saponificación y una mejor separación de fracciones, pero hemos detectado los inconvenientes siguientes: a) el análisis por cromatografía de gases se efectúa con los esteroles libres dando lugar a una descomposición parcial de los esteroles durante la transferencia del cromatógrafo líquido al de gases; b) a veces se observan importantes interferencias positivas en el colesterol; y c) el método sólo es válido para el análisis de esteroles puesto que la metilación no libera totalmente los dialcoholes triterpénicos.

Amelio et al. (1992) describen un método analítico para el análisis simultáneo de ambas familias de compuestos que consiste en el fraccionamiento del insaponificable mediante cromatografía líquida de alta eficacia (CLAE) sobre columna de sílica gel, utilizando gradiente de disolventes y un detector de luz UV a longitud de onda de $210 \mathrm{~nm}$. Los esteroles y dialcoholes triterpénicos se recogen en una sola fracción que se evapora y el residuo se silaniza para el posterior análisis por CG. Este método presenta el inconveniente de utilizar la CLAE con gradiente de disolventes y un detector UV, ambos equipos no habituales en laboratorios dedicados al análisis de grasas.

En el presente trabajo se ensaya la separación de fracciones por CLAE en régimen isocrático, usando un detector de índice de refracción (IR), equipo instrumental que ya se utiliza para el análisis de triglicéridos. Para comparar los resultados con el método oficial y verificar la precisión del método, se han analizado varias muestras por ambos métodos en un solo laboratorio y posteriormente se ha efectuado un análisis colaborativo entre varios laboratorios. Los resultados se han estudiado estadísticamente.

\section{EXPERIMENTAL}

\subsection{Reactivos}

Éter dietílico, etanol, cloroformo, piridina, sulfato sódico anhidro, trimetilclorosilano y hexametildisilazano de pureza analítica. Hexano y éter dietílico de pureza HPLC se usaron para la separación por CLAE.

La potasa alcohólica para la saponificación fue preparada disolviendo $6,5 \mathrm{~g}$ de hidróxido potásico en $10 \mathrm{ml}$ de agua destilada y diluyendo con etanol hasta $50 \mathrm{ml}$.

Se utilizó una solución patrón de $2 \mathrm{mg} / \mathrm{mL}$ de $\alpha$-colestanol ( $5 \alpha$-colestan-3 $\beta$-ol, 95\% GC, Fluka) en cloroformo.

La alúmina desactivada se preparó calentando óxido de aluminio neutro para cromatografía a $105^{\circ} \mathrm{C}$ durante 3 horas y luego se dejó enfriar en un desecador. Una vez a temperatura ambiente se añaden $6 \mathrm{~mL}$ de agua por cada $100 \mathrm{~g}$ de alúmina y se agita durante una hora.

El reactivo de silanización fue piridina-hexametildisilazano-trimetilclorosilano 9:3:1.

\subsection{Materiales y aparatos}

Las experiencias de puesta a punto del método analítico realizadas en el laboratorio de Agra, S.A. se llevaron a cabo como se indica a continuación:

La CCF se realizó sobre placas de vidrio recubiertas de sílica gel 60 , de $0,25 \mathrm{~mm}$ de espesor (Merck) desarrollando dos veces con la mezcla hexano-éter (70:3).

Para la CLAE se utilizó un cromatógrafo de líquidos formado por válvula de inyección (Rheodyne 7125) con un volumen de inyección de $0,2 \mathrm{~mL}$, bomba de líquidos de alta presión (Merck L-600) y detector de índice de refracción (Merck RI-71), usando una columna $(25 \mathrm{~cm} \times 4 \mathrm{~mm}$ de d.i.) rellena de sílica gel 60 de tamaño de partícula $5 \mu \mathrm{m}$ (Merck 50830) y como fase móvil la mezcla hexano-éter etílico 50:50 a un flujo de $1 \mathrm{~mL} / \mathrm{min}$.

La cromatografía de gases se llevó a cabo en un cromatógrafo equipado con un detector de ionización de llama (Hewlett-Packard, HP-5890 Serie II), utilizando una columna de sílice fundida $(25 \mathrm{~m} \times 0,25 \mathrm{~mm}$ de d.i.) impregnada con $5 \%$-fenilmetilsilicona $(0,25 \mu \mathrm{m}$ de espesor) a una temperatura de $265^{\circ} \mathrm{C}$, y como gas portador helio con una presión en cabeza de $100 \mathrm{kPa}$. Las temperaturas del inyector y detector fueron $300 \mathrm{y}$ $310^{\circ} \mathrm{C}$ respectivamente. Se usó un inyector con divisor de flujo con una relación 1:50. Para la obtención de los cromatogramas y cálculo de las áreas se utilizó una estación de proceso de datos Hewlett-Packard. 


\subsection{Métodos}

\subsubsection{Métodos oficiales (separación por CCF)}

Para la determinación de esteroles y de eritrodiol+uvaol se siguieron los procedimientos indicados en los Anexos V y VI del Reglamento CEE/2568/91 de la Comisión de las Comunidades Europeas respectivamente. Etapas comunes para ambos métodos fueron la obtención del insaponificable al éter etílico y su fraccionamiento por CCF. A continuación, en el primer caso se raspa únicamente la banda de los esteroles, mientras que en el segundo se raspan conjuntamente las bandas de esteroles y dialcoholes triterpénicos. Ambas porciones de sílica gel raspada se extraen independientemente con cloroformo y los residuos de la evaporización se silanizan. Finalmente, los productos de reacción se inyectan en el cromatógrafo de gases.

\subsubsection{Método por CLAE}

El aceite $(5 \mathrm{~g})$ se calienta a reflujo con potasa etanólica, se añade agua, el insaponificable se extrae con éter etílico y el extracto etéreo se lava tres veces con agua para eliminar los jabones, todo ello realizado según se indica en el método oficial. La fase etérea se filtra a través de un embudo que contiene papel de filtro con $100 \mathrm{~g}$ de sulfato sódico anhidro y a continuación se lava el sulfato con un poco de éter. La mitad de la solución etérea se agita durante $2 \min$ con $1,25 \mathrm{~g}$ de alúmina desactivada, se filtra a través de papel y se evapora a presión reducida hasta sequedad. El residuo se disuelve en $1 \mathrm{~mL}$ de la mezcla hexano-éter dietílico 50:50 en el caso de aceites de oliva y en 1,5 mL en el caso de aceites de orujo. La solución $(0,2 \mathrm{~mL})$ se inyecta en el cromatógrafo de líquidos. El eluido de la columna desde que aparecen los $\Delta 5$-esteroles hasta que terminan de salir los dialcoholes triterpénicos (aproximadamente desde los 11 a los $24 \mathrm{~min}$ ) se recoge en un matraz en forma de corazón de $25 \mathrm{~mL}$ y se evapora a presión reducida. El residuo se trata con 0,2 $\mathrm{mL}$ de reactivo silanizante y se deja estar durante 15 minutos. Una alícuota de la solución $(1-2 \mu \mathrm{L})$ se inyecta en el cromatógrafo de gases. Los esteroles y dialcoholes triterpénicos se cuantifican respecto al $\alpha$ colestanol utilizado como patrón interno, suponiendo para todos los compuestos el mismo factor de respuesta.

\subsection{Estudio de repetibilidad del método por CLAE}

Una muestra de aceite de oliva se analizó 5 veces como se indica en el apartado 2.3.2 determinándose conjuntamente los esteroles con los dialcoholes triterpénicos.

\subsection{Comparación de los métodos oficial y por CLAE, tanto en la determinación de estero- les como en la determinación conjunta de esteroles y dialcoholes triterpénicos}

Se utilizaron las 4 muestras de aceite siguientes: oliva, oliva con $1 \%$ de girasol, oliva con $2 \%$ de girasol y orujo de oliva con $2 \%$ de girasol. Se obtuvieron las disoluciones del insaponificable por ambos métodos (ver 2.3.1 y 2.3.2) y cada una de ellas se dividió en dos partes iguales. Una alícuota se utilizó para obtener una fracción que sólo contuviese esteroles y la otra para obtener una fracción que contuviese los esteroles junto con los dialcoholes. Por tanto, se obtuvieron por ambos métodos valores de esteroles analizados con y sin la presencia de los dialcoholes.

\subsection{Análisis colaborativo interlaboratorios}

El estudio colaborativo se planteó siguiendo las indicaciones dadas por W. Horwitz (1988). A cada laboratorio participante se le enviaron 8 muestras identificadas con claves que correspondían a duplicados de 2 tipos de aceites de oliva y 2 tipos de aceite de orujo de oliva (tabla I). A las muestras se les añadieron distintos porcentajes de aceite de girasol (normal y variedad alto oleico) para obtener diversas concentraciones de $\Delta 7$-estigmastenol. La remisión de duplicados con código diferente tuvo por objeto evaluar la repetibilidad del método.

Con las muestras se incluía una circular donde se indicaba el procedimiento a seguir, una descripción detallada del procedimiento por CLAE y una hoja de resultados solicitando información del material y de los métodos utilizados en la realización de los análisis. Partiendo de $5 \mathrm{~g}$ de muestra se preparó la solución etérea de insaponificable que se dividió en dos partes: una para realizar el análisis por los métodos oficiales (ver 2.3.1) y otra para aplicar el método de separación por CLAE (ver 2.3.2). En el primer caso, se prepararon y desarrollaron dos placas de CCF; en la primera se raspó únicamente la banda de esteroles y en la segunda la banda de esteroles junto con la de dialcoholes triterpénicos. En el análisis por CLAE se recogió una sola fracción conteniendo ambas familias de compuestos.

Tabla I

Descripción de las muestras

\begin{tabular}{|c|c|c|}
\hline Código & Aceite & Acidez (\%) \\
\hline 1 y 4 & $\begin{array}{l}\text { Orujo de oliva crudo }+5 \% \text { oliva refinado } \\
\qquad+3 \% \text { girasol } \mathrm{AO}\end{array}$ & 10.8 \\
\hline 2 y 8 & Oliva refinado $+1 \%$ girasol $\mathrm{AO}$ & 0.41 \\
\hline 3 y 7 & Oliva lampante $+1 \%$ girasol $A O$ & 3.8 \\
\hline 5 y 6 & $\begin{array}{l}\text { Orujo de oliva refinado }+1,5 \% \text { girasol } \\
\text { refinado }\end{array}$ & 0.20 \\
\hline
\end{tabular}


El análisis estadístico de repetibilidad de los resultados se realizó de acuerdo con la Norma Internacional ISO 5275/1986 (E) donde se indican los criterios usados tanto para el rechazo de resultados como los procedimientos matemáticos seguidos. Como criterio de verificación de resultados anómalos se usaron los métodos de Cochran y Dixon, determinando respectivamente laboratorios que dan resultados cuya diferencia entre valores es excepcionalmente alta y laboratorios que dan valores que son significativamente diferentes a los aportados por los demás:

Los parámetros estadísticos utilizados fueron los siguientes:

$S_{r}$ : Desviación estándar de la repetibilidad

$r$ : Repetibilidad $\left(2.8 \sqrt{S_{r}^{2}}\right)$.

$R S D_{r}$ : Desviación estándar de la repetibilidad.

$S_{r}$ : Desviación estándar de la reproducibilidad.

$R$ : Reproducibilidad $\left(2.8 \sqrt{S_{R}^{2}}\right)$.

RSDR: Desviación estándar relativa de la reproducibilidad.

$$
\begin{aligned}
& H o_{R}: \text { Indice Horrat }=\left[\frac{R S D_{R \exp }}{R S D_{R \text { teor }}}\right] \\
& \text { donde } R S D_{R \text { teor }}=2^{(1-0.5 \log \mathrm{C})}
\end{aligned}
$$

siendo $\mathrm{C}$ la concentración del analito expresada en potencias de 10.

De entre estos parámetros es importante destacar el significado de la repetibilidad que indica que los valores obtenidos en dos determinaciones sucesivas realizadas sobre la misma muestra, utilizando el mismo método analítico, no diferirán en más del valor de $r$. De la misma manera, la reproducibilidad expresa que los valores obtenidos por dos laboratorios diferentes sobre una misma muestra, utilizando el mismo método analítico, no diferirán en más del valor de $R$. También cabe destacar el significado del índice Horrat (Horowitz ratio) que tiene en cuenta el orden de magnitud de la concentración del analito. Se calcula mediante el cociente entre la RSDR experimental y la teórica, de tal manera que valores iguales o menores que 1 indican que el método analítico tiene una buena reproducibilidad (Pocklington, 1991).

\subsection{Método estadístico para la comparación de resultados}

Para la comparación de los resultados de las determinaciones realizadas por diversos métodos analíticos se realizó un análisis estadístico de varianza mediante el programa informático Statistica (StatSoft Inc), utilizando el test ANOVA de dos direcciones.

\section{RESULTADOS Y DISCUSIÓN}

\subsection{Determinación por CLAE}

En el cromatograma obtenido por CLAE con detector de índice de refracción del insaponificable de un aceite de girasol (figura 1) se observa que la fracción esterólica se desdobla en tres picos correspondientes a los $\Delta 5$-esteroles, $a$-colestanol y $\Delta 7$-esteroles, quedando perfectamente separada de la fracción de alcoholes triterpénicos. En el cromatograma de un aceite de oliva (figura 2) el pico correspondiente a los $\Delta 7$-esteroles es muy pequeño y aparece el correspondiente a los dialcoholes triterpénicos, sin que entre ambos se observe la presencia de otro tipo de compuestos. Por tanto la fracción que eluye desde un poco antes de los esteroles hasta un poco después de los dialcoholes contiene casi exclusivamente estos compuestos. El análisis por CG de los derivados sililados origina un cromatograma prácticamente libre de interferencias (figura 3 ).

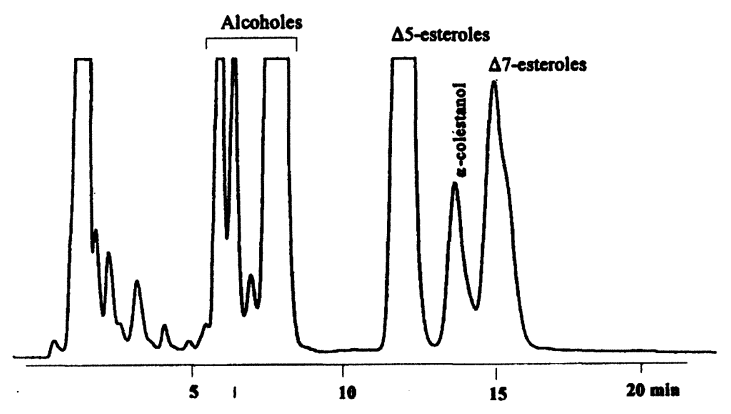

Figura 1

Cromatograma de CLAE con detector de índice de refracción del insaponificable de un aceite de girasol refinado.

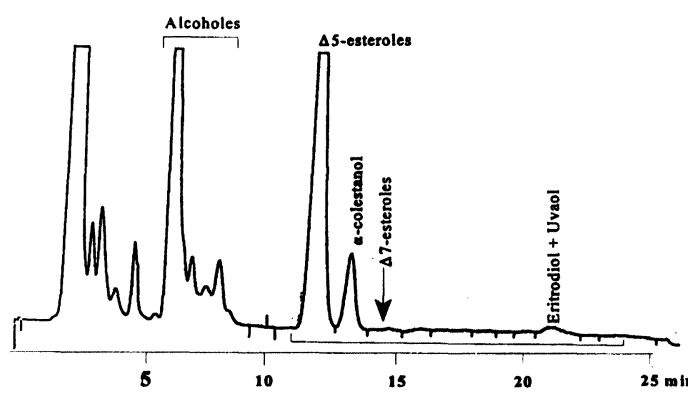

Figura 2

Cromatograma de CLAE con detector del índice de refracción del insaponificable de un aceite de oliva refinado. 


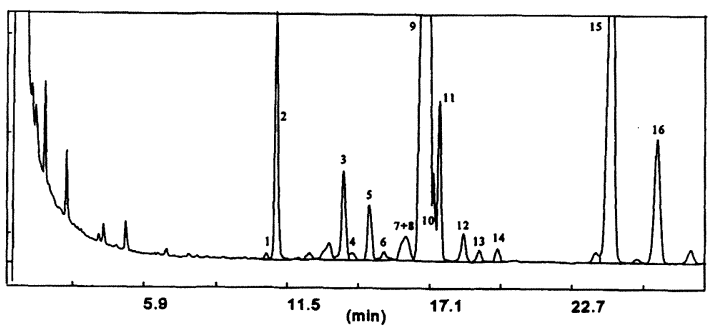

Figura 3

Cromatograma de gases de la fracción esteroles + dialcoholes obtenida por fraccionamiento por CLAE de un insaponificable de aceite de orujo de oliva refinado. 1. colesterol; 2 . a-colestanol; 3. campesterol; 4. campestanol; 5. estigmasterol; 6 . $\Delta 7$ campesterol; 7. $\Delta 5,23$-estigmastadienol; 8. clerosterol; 9 . $\beta$ sitosterol; 10. sitostanol; 11. $\Delta 5$-avenasterol; 12. $\Delta 5$, 24estigmastadienol; 13. $\Delta 7$-estigmastenol; 14. $\Delta 7$-avenasterol; 15.

\subsection{Repetibilidad del método CLAE-esteroles- dialcoholes}

Los resultados de las 5 determinaciones realizadas en un solo laboratorio sobre una muestra de aceite de oliva, mediante el procedimiento que utiliza separación de fracciones por CLAE, se indican en la Tabla II. Puede observarse que la repetibilidad es buena en todos los casos excepto en el colesterol. Esta falta de precisión en la medida del colesterol está de acuerdo con la existencia de impurezas en la banda de dialcoholes que en la cromatografía de gases aparecen en la zona del colesterol (León y Cert, 1994).

\subsection{Comparación de métodos}

El análisis estadístico de los resultados obtenidos en el análisis de cuatro muestras en un solo laboratorio no mostró diferencias significativas entre los valores de colesterol, brasicasterol, 24-metilencolesterol, campesterol, campestanol, estigmasterol y esteroles totales obtenidos por los cuatro métodos objeto de la comparación: CCF-esteroles, CCF-esteroles + dialcoholes, CLAE-esteroles y CLAE-esteroles + dialcoholes. Tampoco se encontraron diferencias entre los valores de eritrodiol + uvaol obtenidos por CCF-esteroles + dialcoholes y CLAE-esteroles + dialcoholes.

Los valores de $\beta$-sitosterol aparente, $\Delta 7$-estigmastenol y $\Delta 7$-avenasterol tampoco mostraron diferencias en las determinaciones por CLAE-esteroles y CLAE-esteroles + dialcoholes, lo cual hace posible las determinaciones de esteroles y dialcoholes en un solo análisis cromatográfico. Sin embargo el método CLAE-esteroles + dialcoholes en comparación con el CCF-esteroles + dialcoholes da lugar a una disminución de los valores de $\Delta 7$ estigmastenol (valor medio del $0,18 \%$ ) con un nivel de significación del $96 \%$. Este hecho sugiere que la CLAE elimina la interferencia positiva que a veces tiene lugar cuando se efectúa la separación por CCF, probablemente debida al cicloartenol, compuesto que en CCF no se separa perfectamente de los esteroles, o debida a compuestos minoritarios que en CCF eluyen con el eritrodiol.

La comparación del método CCF-esteroles (método oficial) con los otros tres muestra unas diferencias entre los valores de estos esteroles con altos niveles de significación (Tabla III). Se observa que los tres métodos dan lugar a valores de los $\Delta 7$-esteroles mayores que el méto-

Tabla II

Repetibilidad de la determinación de esteroles y dialcoholes triterpénicos por el método de separación por CLAE

\begin{tabular}{lcccccccc}
\hline & $\begin{array}{c}\text { Colesterol } \\
(\%)\end{array}$ & $\begin{array}{c}\text { Campesterol } \\
(\%)\end{array}$ & $\begin{array}{c}\text { Estigmasterol } \\
(\%)\end{array}$ & $\begin{array}{c}\text { B-sitosterol } \\
\text { aparente } \\
(\%)\end{array}$ & $\begin{array}{c}\Delta 7 \text {-estigmastenol } \\
(\%)\end{array}$ & $\begin{array}{c}\Delta 7 \text {-avenasterol } \\
(\%)\end{array}$ & $\begin{array}{c}\text { Esteroles } \\
\text { totales } \\
(\mathrm{mg} / \mathrm{Kg})\end{array}$ & $\begin{array}{c}\text { Eritrodiol + } \\
\text { uvaol }(\%)\end{array}$ \\
\hline Ensayo 1 & 0,15 & 3,26 & 0,85 & 94,5 & 0,49 & 0,32 & 1292 & 3,27 \\
Ensayo 2 & 0,14 & 3,28 & 0,84 & 94,4 & 0,47 & 0,33 & 1232 & 3,37 \\
Ensayo 3 & 0,13 & 3,59 & 0,83 & 94,3 & 0,46 & 0,27 & 1290 & 3,00 \\
Ensayo 4 & 0,20 & 3,32 & 0,89 & 94,7 & 0,46 & 0,31 & 1272 & 3,20 \\
Ensayo 5 & 0,26 & 3,39 & 0,85 & 94,7 & 0,47 & 0,29 & 1221 & 3,10 \\
\hline Media & 0,16 & 3,37 & 0,85 & 94,5 & 0,47 & 0,30 & 1261 & 3,19 \\
$S D_{r}$ & 0,034 & 0,134 & 0,023 & 0,181 & 0,012 & 0,024 & 33,03 & 0,144 \\
$R S D_{r}(\%)$ & 20,7 & 3,97 & 2,68 & 0,19 & 2,60 & 7,89 & 2,62 & 4,52 \\
$r(95 \%)^{a}$ & 0,094 & 0,372 & 0,063 & 0,503 & 0,034 & 0,067 & 91,8 & 0,400 \\
\hline
\end{tabular}

(a) $r(95 \%)=$ repetibilidad al $95 \%$ de confianza $\left(t_{95, v=4} \times S D\right)$. 
Tabla III

Diferencias medias y niveles de significación estadística de los resultados de la determinación por el método CCF-esteroles respecto a las medidas obtenidas por otros métodos

\begin{tabular}{|c|c|c|c|c|c|c|}
\hline \multirow{3}{*}{ Esteroles } & \multicolumn{6}{|c|}{ Método analítico } \\
\hline & \multicolumn{2}{|c|}{$\begin{array}{c}\text { CCF } \\
\text { esteroles + dialcoholes }\end{array}$} & \multicolumn{2}{|c|}{$\begin{array}{c}\text { CLAE } \\
\text { esteroles }\end{array}$} & \multicolumn{2}{|c|}{$\begin{array}{c}\text { CLAE } \\
\text { esteroles + dialcoholes }\end{array}$} \\
\hline & $d a(\%)$ & n.c.b & $d(\%)$ & n.c. & $d(\%)$ & n.c. \\
\hline$\beta$-sitosterol & $-1,01$ & 97,1 & $-0,32$ & 86,1 & $-0,43$ & 96,7 \\
\hline$\Delta 7$-estigmastenol & $+0,34$ & 96,6 & $+0,16$ & 94,5 & $+0,13$ & 94,5 \\
\hline$\Delta 7$-avenasterol & $+0,27$ & 99,4 & $+0,32$ & 99,5 & $+0,29$ & 99,8 \\
\hline
\end{tabular}

(a) Diferencia entre las medias de los valores obtenidos para las cuatro muestras mediante el método analítico indicado y el método CCF-esteroles. (b) Nivel de confianza estadística expresado en \%.

do oficial (CCF-esteroles) como consecuencia de la pérdida que se produce en este último al raspar únicamente la banda de esteroles en la CCF (León y Cert, 1994). Esta pérdida también justifica una parte de la disminución que se observa en el $\beta$-sitosterol aparente.

\subsection{Análisis colaborativo interlaboratorios de la determinación de esteroles}

A la vista de la similitud de resultados entre los métodos CLAE-esteroles y CLAE-esteroles + dialcoholes, se decidió organizar un estudio colaborativo de determina-

Tabla IV

Parámetros estadísticos de la determinación de colesterol

\begin{tabular}{|c|c|c|c|c|c|c|c|c|}
\hline \multirow{2}{*}{$\begin{array}{c}\text { Muestras } \\
\text { Método }\end{array}$} & \multicolumn{2}{|c|}{ Orujo crudo } & \multicolumn{2}{|c|}{ Oliva refinado } & \multicolumn{2}{|c|}{ Oliva lampante } & \multicolumn{2}{|c|}{ Orujo refinado } \\
\hline & CCFa & CLAEb & CCFa & CLAEb & CCFa & CLAEb & CCFa & CLAEb \\
\hline $\begin{array}{c}\text { Número } \\
\text { participantes }\end{array}$ & 17 & 11 & 17 & 11 & 17 & 11 & 17 & 11 \\
\hline $\begin{array}{l}\text { Laboratorios } \\
\text { rechazados }\end{array}$ & 3 & 1 & 2 & 2 & 1 & 0 & 0 & 1 \\
\hline Valor medio (\%) & 0,19 & 0,17 & 0,21 & 0,15 & 0,18 & 0,18 & 0,23 & 0,20 \\
\hline \multicolumn{9}{|l|}{ Repetibilidad } \\
\hline$S_{r}$ & 0,04 & 0,03 & 0,07 & 0,02 & 0,05 & 0,06 & 0,07 & 0,02 \\
\hline$r$ & 0,11 & 0,08 & 0,19 & 0,15 & 0,13 & 0,16 & 0,20 & 0,62 \\
\hline$R D S_{r}(\%)$ & 19,8 & 17,0 & 33,3 & 13,3 & 25,4 & 31,4 & 29,9 & 10,9 \\
\hline \multicolumn{9}{|l|}{ Reproducibilidad } \\
\hline$S_{R}$ & 0,06 & 0,07 & 0,11 & 0,07 & 0,12 & 0,08 & 0,13 & 0,09 \\
\hline$R$ & 0,16 & 0,19 & 0,29 & 0,20 & 0,33 & 0,23 & 0,37 & 0,26 \\
\hline$R S D_{R}(\%)$ & 30,1 & 41,6 & 50,5 & 48,1 & 66,0 & 44,9 & 57,2 & 46,0 \\
\hline $\mathrm{Ho}_{\mathrm{R}}$ & 1,5 & 2,0 & 2,5 & 2,3 & 3,2 & 2,2 & 2,9 & 2,3 \\
\hline
\end{tabular}

a Cromatografía de Capa Fina de Esteroles

b Cromatografía Líquida de Alta Eficacia de Esteroles y Dialcoholes 
Tabla V

Parámetros estadísticos de la determinación de campesterol

\begin{tabular}{|c|c|c|c|c|c|c|c|c|}
\hline \multirow{2}{*}{$\begin{array}{c}\text { Muestras } \\
\text { Método }\end{array}$} & \multicolumn{2}{|c|}{ Orujo crudo } & \multicolumn{2}{|c|}{ Oliva refinado } & \multicolumn{2}{|c|}{ Oliva lampante } & \multicolumn{2}{|c|}{ Orujo refinado } \\
\hline & CCFa & CLAEb & CCFa & CLAEb & CCFa & CLAEb & CCFa & CLAEb \\
\hline $\begin{array}{c}\text { Número } \\
\text { participantes }\end{array}$ & 17 & 12 & 17 & 12 & 17 & 12 & 17 & 12 \\
\hline $\begin{array}{l}\text { Laboratorios } \\
\text { rechazados }\end{array}$ & 1 & 2 & 0 & 2 & 0 & 2 & 2 & 1 \\
\hline Valor medio (\%) & 3,29 & 3,29 & 2,94 & 2,91 & 3,00 & 2,94 & 3,38 & 3,32 \\
\hline \multicolumn{9}{|l|}{ Repetibilidad } \\
\hline$S_{r}$ & 0,08 & 0,09 & 0,10 & 0,05 & 0,10 & 0,04 & 0,08 & 0,11 \\
\hline$r$ & 0,22 & 0,26 & 0,29 & 0,14 & 0,30 & 0,12 & 0,23 & 0,30 \\
\hline$R D S_{r}(\%)$ & 2,3 & 2,9 & 3,5 & 1,7 & 3,5 & 1,4 & 2,4 & 3,2 \\
\hline \multicolumn{9}{|l|}{ Reproducibilidad } \\
\hline$S_{R}$ & 0,16 & 0,21 & 0,15 & 0,15 & 0,19 & 0,10 & 0,13 & 0,18 \\
\hline$R$ & 0,46 & 0,60 & 0,42 & 0,42 & 0,54 & 0,29 & 0,36 & 0,51 \\
\hline$R S D_{R}(\%)$ & 5,0 & 6,5 & 5,1 & 5,2 & 6,4 & 3,5 & 3,8 & 5,4 \\
\hline$H o_{R}$ & 0,4 & 0,5 & 0,4 & 0,4 & 0,5 & 0,3 & 0,3 & 0,4 \\
\hline
\end{tabular}

Tabla VI

Parámetros estadísticos de la determinación de estigmasterol

\begin{tabular}{|c|c|c|c|c|c|c|c|c|}
\hline \multirow{2}{*}{$\begin{array}{c}\text { Muestras } \\
\text { Método }\end{array}$} & \multicolumn{2}{|c|}{ Orujo Crudo } & \multicolumn{2}{|c|}{ Oliva Refinado } & \multicolumn{2}{|c|}{ Oliva lampante } & \multicolumn{2}{|c|}{ Orujo refinado } \\
\hline & CCFa & $\mathrm{CLAE}^{\mathrm{b}}$ & CCFa & CLAEb & CCFa & CLAEb & CCFa & CLAEb $^{b}$ \\
\hline $\begin{array}{c}\text { Número } \\
\text { participantes }\end{array}$ & 17 & 12 & 17 & 12 & 17 & 12 & 17 & 12 \\
\hline $\begin{array}{l}\text { Laboratorios } \\
\text { rechazados }\end{array}$ & 2 & 3 & 2 & 1 & 3 & 1 & 2 & 2 \\
\hline Valor medio (\%) & 1,59 & 1,56 & 1,20 & 1,14 & 1,31 & 1,24 & 1,46 & 1,40 \\
\hline \multicolumn{9}{|l|}{ Repetibilidad } \\
\hline$S_{r}$ & 0,04 & 0,05 & 0,04 & 0,07 & 0,05 & 0,06 & 0,05 & 0,02 \\
\hline$r$ & 0,10 & 0,14 & 0,11 & 0,19 & 0,13 & 0,18 & 0,14 & 0,06 \\
\hline$R D S_{r}(\%)$ & 2,2 & 3,3 & 3,2 & 5,9 & 3,4 & 5,2 & 3,4 & 1,6 \\
\hline \multicolumn{9}{|l|}{ Reproducibilidad } \\
\hline$S_{R}$ & 0,08 & 0,08 & 0,10 & 0,10 & 0,10 & 0,14 & 0,07 & 0,07 \\
\hline$R$ & 0,22 & 0,23 & 0,27 & 0,28 & 0,27 & 0,39 & 0,20 & 0,21 \\
\hline$R S D_{R}(\%)$ & 5,0 & 5,4 & 8,1 & 8,9 & 7,5 & 11,2 & 5,0 & 5,4 \\
\hline $\mathrm{Ho}_{\mathrm{R}}$ & 0,3 & 0,4 & 0,5 & 0,6 & 0,5 & 0,7 & 0,3 & 0,4 \\
\hline
\end{tabular}


Tabla VII

Parámetros estadísticos de la determinación de $\beta$-Sitosterol aparente

\begin{tabular}{|c|c|c|c|c|c|c|c|c|}
\hline \multirow{2}{*}{$\begin{array}{c}\text { Muestras } \\
\text { Método }\end{array}$} & \multicolumn{2}{|c|}{ Orujo crudo } & \multicolumn{2}{|c|}{ Oliva refinado } & \multicolumn{2}{|c|}{ Oliva lampante } & \multicolumn{2}{|c|}{ Orujo refinado } \\
\hline & CCFa & CLAEb & CCFa & CLAEb & CCFa & CLAEb & CCFa & CLAEb \\
\hline $\begin{array}{c}\text { Número } \\
\text { participantes }\end{array}$ & 17 & 12 & 17 & 12 & 17 & 12 & 17 & 12 \\
\hline $\begin{array}{l}\text { Laboratorios } \\
\text { rechazados }\end{array}$ & 2 & 1 & 3 & 2 & 1 & 2 & 1 & 3 \\
\hline Valor medio (\%) & 93,44 & 93,07 & 94,29 & 94,22 & 94,10 & 94,00 & 93,05 & 92,61 \\
\hline \multicolumn{9}{|l|}{ Repetibilidad } \\
\hline$S_{r}$ & 0,28 & 0,38 & 0,15 & 0,15 & 0,23 & 0,19 & 0,30 & 0,16 \\
\hline$r$ & 0,79 & 1,07 & 0,43 & 0,42 & 0,66 & 0,54 & 0,85 & 0,45 \\
\hline$R D S_{r}(\%)$ & 0,30 & 0,41 & 0,16 & 0,16 & 0,25 & 0,21 & 0,33 & 0,17 \\
\hline \multicolumn{9}{|l|}{ Reproducibilidad } \\
\hline$S_{R}$ & 0,52 & 0,60 & 0,38 & 0,28 & 0,49 & 0,37 & 0,62 & 0,42 \\
\hline$R$ & 1,46 & 1,69 & 1,06 & 0,79 & 1,38 & 1,03 & 1,73 & 1,18 \\
\hline$R S D_{R}(\%)$ & 0,56 & 0,65 & 0,40 & 0,30 & 0,52 & 0,39 & 0,66 & 0,46 \\
\hline $\mathrm{Ho}_{\mathrm{R}}$ & 0,07 & 0,08 & 0,05 & 0,04 & 0,06 & 0,05 & 0,08 & 0,06 \\
\hline
\end{tabular}

a Cromatografía de Capa Fina de Esteroles

b Cromatografía Líquida de Alta Eficacia de Esteroles y Dialcoholes

Tabla VIII

Parámetros estadísticos de la determinación de $\Delta 7$-Estigmastenol

\begin{tabular}{|c|c|c|c|c|c|c|c|c|}
\hline \multirow{2}{*}{$\begin{array}{c}\text { Muestras } \\
\text { Método }\end{array}$} & \multicolumn{2}{|c|}{ Orujo crudo } & \multicolumn{2}{|c|}{ Oliva refinado } & \multicolumn{2}{|c|}{ Oliva lampante } & \multicolumn{2}{|c|}{ Orujo refinado } \\
\hline & CCFa & CLAEb & CCFa & CLAEb $^{b}$ & CCFa & CLAEb $^{b}$ & CCFa & CLAEb \\
\hline $\begin{array}{c}\text { Número } \\
\text { participantes }\end{array}$ & 17 & 12 & 17 & 12 & 17 & 12 & 17 & 12 \\
\hline $\begin{array}{l}\text { Laboratorios } \\
\text { rechazados }\end{array}$ & 1 & 1 & 2 & 0 & 1 & 2 & 0 & 1 \\
\hline Valor medio (\%) & 0,55 & 0,69 & 0,42 & 0,49 & 0,38 & 0,44 & 0,66 & 0,82 \\
\hline \multicolumn{9}{|l|}{ Repetibilidad } \\
\hline$S_{r}$ & 0,06 & 0,08 & 0,07 & 0,06 & 0,06 & 0,04 & 0,08 & 0,08 \\
\hline$r$ & 0,16 & 0,22 & 0,19 & 0,18 & 0,16 & 0,12 & 0,23 & 0,22 \\
\hline$R D S_{r}(\%)$ & 10,2 & 11,4 & 16,0 & 12,8 & 15,2 & 10,1 & 12,2 & 9,6 \\
\hline \multicolumn{9}{|l|}{ Reproducibilidad } \\
\hline$S_{R}$ & 0,18 & 0,12 & 0,09 & 0,09 & 0,09 & 0,06 & 0,16 & 0,15 \\
\hline$R$ & 0,50 & 0,33 & 0,25 & 0,26 & 0,26 & 0,16 & 0,45 & 0,42 \\
\hline$R S D_{R}(\%)$ & 32,9 & 17,4 & 21,0 & 19,2 & 25,0 & 12,9 & 24,4 & 18,3 \\
\hline $\mathrm{Ho}_{\mathrm{R}}$ & 1,9 & 1,0 & 1,2 & 1,1 & 1,3 & 0,7 & 1,4 & 1,1 \\
\hline
\end{tabular}


Tabla IX

Parámetros estadísticos de la determinación de $\Delta 7$-Avenasterol

\begin{tabular}{|c|c|c|c|c|c|c|c|c|}
\hline \multirow{2}{*}{$\begin{array}{c}\text { Muestras } \\
\text { Método }\end{array}$} & \multicolumn{2}{|c|}{ Orujo crudo } & \multicolumn{2}{|c|}{ Oliva refinado } & \multicolumn{2}{|c|}{ Oliva lampante } & \multicolumn{2}{|c|}{ Orujo refinado } \\
\hline & CCFa & CLAEb & CCFa & CLAEb & CCFa & CLAEb & CCFa & CLAEb \\
\hline $\begin{array}{c}\text { Número } \\
\text { participantes }\end{array}$ & 17 & 12 & 17 & 12 & 17 & 12 & 17 & 12 \\
\hline $\begin{array}{l}\text { Laboratorios } \\
\text { rechazados }\end{array}$ & 0 & 2 & 1 & 1 & 0 & 4 & 1 & 1 \\
\hline Valor medio $(\mathrm{mg} / \mathrm{Kg})$ & 0,41 & 0,48 & 0,50 & 0,59 & 0,47 & 0,57 & 0,41 & 0,49 \\
\hline \multicolumn{9}{|l|}{ Repetibilidad } \\
\hline$S_{r}$ & 0,10 & 0,04 & 0,05 & 0,03 & 0,11 & 0,02 & 0,08 & 0,04 \\
\hline$r$ & 0,28 & 0,11 & 0,15 & 0,08 & 0,32 & 0,06 & 0,22 & 0,12 \\
\hline$R D S_{r}(\%)$ & 25,0 & 8,4 & 10,4 & 4,7 & 24,2 & 3,6 & 18,9 & 8,8 \\
\hline \multicolumn{9}{|l|}{ Reproducibilidad } \\
\hline$S_{R}$ & 0,20 & 0,13 & 0,18 & 0,12 & 0,13 & 0,03 & 0,16 & 0,12 \\
\hline$R$ & 0,55 & 0,36 & 0,52 & 0,33 & 0,38 & 0,08 & 0,45 & 0,32 \\
\hline$R S D_{R}(\%)$ & 48,5 & 26,5 & 36,8 & 20,1 & 28,7 & 5,0 & 39,1 & 23,4 \\
\hline $\mathrm{Ho}_{\mathrm{R}}$ & 2,6 & 1,5 & 2,1 & 1,2 & 1,6 & 0,3 & 2,1 & 1,3 \\
\hline
\end{tabular}

Tabla X

Parámetros estadísticos de la determinación de esteroles totales

\begin{tabular}{|c|c|c|c|c|c|c|c|c|}
\hline \multirow{2}{*}{$\begin{array}{c}\text { Muestras } \\
\text { Método }\end{array}$} & \multicolumn{2}{|c|}{ Orujo crudo } & \multicolumn{2}{|c|}{ Oliva refinado } & \multicolumn{2}{|c|}{ Oliva lampante } & \multicolumn{2}{|c|}{ Orujo Refinado } \\
\hline & CCFa & $\mathrm{CLAE}^{\mathrm{b}}$ & CCFa & CLAE $^{b}$ & CCFa & CLAEb & CCFa & CLAEb \\
\hline \multicolumn{9}{|l|}{ Número } \\
\hline participantes & 15 & 10 & 15 & 10 & 16 & 10 & 15 & 10 \\
\hline \multicolumn{9}{|l|}{$\begin{array}{l}\text { Laboratorios } \\
\text { rechazados }\end{array}$} \\
\hline Valor medio $(\mathrm{mg} / \mathrm{Kg})$ & $\begin{array}{r}3 \\
3668\end{array}$ & $\begin{array}{r}1 \\
3547\end{array}$ & $\begin{array}{r}3 \\
1845\end{array}$ & $\begin{array}{r}2 \\
1813\end{array}$ & $\begin{array}{r}0 \\
1844\end{array}$ & $\begin{array}{r}0 \\
1737\end{array}$ & $\begin{array}{r}2 \\
2576\end{array}$ & $\begin{array}{r}1 \\
2409\end{array}$ \\
\hline \multicolumn{9}{|l|}{ Repetibilidad } \\
\hline$S_{r}$ & 208 & 177 & 49 & 27 & 158 & 102 & 93 & 38 \\
\hline$r$ & 582 & 495 & 138 & 77 & 442 & 286 & 260 & 106 \\
\hline$R D S_{r}(\%)$ & 5,70 & 5,0 & 2,7 & 1,5 & 8,6 & 5,9 & 3,6 & 1,6 \\
\hline \multicolumn{9}{|l|}{ Reproducibilidad } \\
\hline$S_{R}$ & 290 & 226 & 154 & 164 & 215 & 158 & 297 & 135 \\
\hline$R$ & 813 & 633 & 431 & 459 & 601 & 442 & 831 & 379 \\
\hline$R S D_{R}(\%)$ & 7,9 & 6,4 & 8,3 & 9,0 & 11,6 & 9,1 & 11,5 & 5,6 \\
\hline$H o_{R}$ & 1,7 & 1,4 & 1,6 & 1,7 & 2,3 & 1,7 & 2,3 & 1,1 \\
\hline
\end{tabular}

a Cromatografía de Capa Fina de Esteroles

b Cromatografía Líquida de Alta Eficacia de Esteroles y Dialcoholes 
ción de esteroles comparando únicamente los métodos oficial (CCF-esteroles) y por CLAE-esteroles + dialcoholes. En el análisis circular participaron 18 laboratorios de la industria y centros oficiales, de los cuales sólo 12 aportaron resultados del método por CLAE. Entre estos últimos, un laboratorio llevó a cabo la purificación del insaponificable mediante lavados alternativos con solución acuosa de hidróxido potásico $0,5 \mathrm{~N}$ y agua en vez de agitar con alúmina, y dos laboratorios utilizaron detector UV a $210 \mathrm{~nm}$ en vez del detector IR, para visualizar la separación por CLAE. La purificación del insaponificable mediante lavados con potasa es un procedimiento también utilizado para eliminar los ácidos grasos. La utilización de un detector UV no afecta a la separación de la fracción esterólica y se ha utilizado en un método similar (Amelio et al., 1995). Por otra parte, los resultados de los tres laboratorios fueron similares a los del resto de participantes. En consecuencia se decidió incluir todos los datos recibidos en el estudio estadístico, cuyos resultados se exponen en las Tablas IV-X.

Los resultados rechazados por la falta de repetibilidad o reproducibilidad al no cumplir los test correspondientes fueron similares en ambos métodos. Comparando la repetibilidad y reproducibilidad medias del método oficial (CCF-esteroles) obtenidas en este estudio, del mismo método en un colaborativo anterior (Moreda et al., 1995) y del método CLAE-esteroles + dialcoholes, no se observan diferencias importantes en la precisión de las determinaciones de colesterol, campesterol, $\beta$-sitosterol aparente y esteroles totales. En cuanto a los restantes esteroles (Tabla XI) se observa lo siguiente: el estigmasterol se determina con mejor preci-

Tabla XI

Comparación de los parámetros de precisión de dos métodos analíticos para la determinación de esteroles

\begin{tabular}{|c|c|c|c|c|}
\hline \multirow[b]{2}{*}{ Esteroles } & \multirow[b]{2}{*}{ Precisión } & \multicolumn{3}{|c|}{ Método analítico } \\
\hline & & $\begin{array}{l}\text { CCF- } \\
\text { esteroles } \\
\text { (Moreda } \\
\text { et al.) }\end{array}$ & $\begin{array}{c}\text { CCF- } \\
\text { esteroles } \\
\text { (estudio } \\
\text { actual) }\end{array}$ & $\begin{array}{c}\text { CLAE } \\
\text { esteroles } \\
+ \\
\text { dialcoholes } \\
\end{array}$ \\
\hline \multirow[t]{2}{*}{ Estigmasterol } & $R S D_{r}(\%)^{\mathrm{a}}$ & 8,1 & 3,1 & 4,0 \\
\hline & $R S D_{R}(\%)^{\mathrm{b}}$ & 10,4 & 6,4 & 7,7 \\
\hline \multirow[t]{2}{*}{$\Delta 7$-estigmastenol } & $R S D_{r}(\%)$ & 19,6 & 13,4 & 11,6 \\
\hline & $R S D_{R}(\%)$ & 32,5 & 25,8 & 17,0 \\
\hline \multirow[t]{2}{*}{$\Delta 7$-avenasterol } & $R S D_{r}(\%)$ & - & 19,6 & 6,4 \\
\hline & $R S D_{R}(\%)$ & - & 38,3 & 18,8 \\
\hline
\end{tabular}

(a) Valor medio de la repetibilidad de las determinaciones realizadas en cada estudio colaborativo.

(b) Valor medio de la reproducibilidad de las determinaciones realizadas en cada estudio colaborativo. sión en los dos métodos ensayados en este estudio con respecto al anterior análisis circular; el $\Delta 7$-estigmastenol se determina con una mejora de la precisión en el actual análisis circular por CCF-esteroles respecto al anterior estudio, mejora que es más acusada en cuanto a reproducibilidad en el método por CLAE; finalmente, el $\Delta 7$ avenasterol muestra una mejora de ambos parámetros de precisión en la determinación por el método CLAE.

La comparación de las medias de los valores medios obtenidos por cada método (Tabla XII) muestra que hay un incremento del $\Delta 7$-estigmastenol y $\Delta 7$-avenasterol con alto nivel de significación y que en relación al valor absoluto de la medida tiene considerable importancia. Este resultado concuerda con lo observado en el experimento de comparación llevado a cabo en el laboratorio (ver Tabla III). También se observa una disminución de los esteroles totales que puede atribuirse a pérdida del patrón a-colestanol en la separación por CCF. Las restantes variables muestran diferencias de escasa entidad.

Tabla XII

Comparación de los valores medios de esteroles obtenidos por el método CLAE-esteroles + dialcoholes y CCF-esteroles en el análisis colaborativo

\begin{tabular}{lcccc}
\hline & \multicolumn{2}{c}{ Método } & & \\
\cline { 2 - 3 } Esteroles & $\begin{array}{c}\text { CLAE } \\
\text { esteroles }+ \\
\text { dialcoholes }\end{array}$ & $\begin{array}{c}\text { CCF } \\
\text { esteroles }\end{array}$ & $\begin{array}{c}\text { diferencia } \\
\text { media }\end{array}$ & $\begin{array}{c}\text { nivel de } \\
\text { confiaza } \\
(\%)\end{array}$ \\
\cline { 2 - 3 } & media & media & & \\
\hline Colesterol (\%) & 0,18 & 0,20 & $-0,02$ & 88,6 \\
Campesterol (\%) & 3,12 & 3,15 & $-0,03$ & 92,0 \\
Estigmasterol (\%) & 1,34 & 1,39 & $-0,05$ & 99,2 \\
$\beta$ B-sitosterol aparente (\%) & 93,47 & 93,72 & $-0,25$ & 92,0 \\
$\Delta 7$-estigmastenol (\%) & 0,61 & 0,50 & $+0,11$ & 97,7 \\
$\Delta 7$-avenasterol (\%) & 0,53 & 0,45 & $+0,08$ & 99,9 \\
Esteroles totales (mg/Kg) & 2376 & 2483 & -107 & 96,8 \\
\hline
\end{tabular}

(a) Media de los valores obtenidos para las cuatro muestras. (b) Diferencia entre las medias CLAE-esteroles + dialcoholes y CCF-esteroles.

\subsection{Estudio colaborativo interlaboratorios de la determinación de eritrodiol + uvaol}

Para la determinación de eritrodiol + uvaol se utilizaron los métodos oficial (CCF-esteroles + dialcoholes) y por CLAE-esteroles + dialcoholes, siguiendo el mismo protocolo que el empleado para los esteroles. Los resultados expuestos en la Tabla XIII indican una precisión similar para ambos métodos. No se encontraron diferencias significativas entre los valores medios obtenidos por cada método. 
Tabla XIII

Parámetros estadísticos de la determinación de Eritrodiol + Uvaol

\begin{tabular}{|c|c|c|c|c|c|c|c|c|}
\hline \multirow{2}{*}{$\begin{array}{c}\text { Muestras } \\
\text { Método }\end{array}$} & \multicolumn{2}{|c|}{ Orujo crudo } & \multicolumn{2}{|c|}{ Oliva refinado } & \multicolumn{2}{|c|}{ Oliva lampante } & \multicolumn{2}{|c|}{ Orujo refinado } \\
\hline & CCFa & CLAE $^{b}$ & CCFa & CLAEb & CCFa & $\mathrm{CLAE}^{\mathrm{b}}$ & CCFa & CLAE $^{b}$ \\
\hline $\begin{array}{c}\text { Número } \\
\text { participantes }\end{array}$ & 17 & 12 & 17 & 12 & 17 & 12 & 17 & 12 \\
\hline $\begin{array}{l}\text { Laboratorios } \\
\text { rechazados }\end{array}$ & 0 & 1 & 3 & 0 & 4 & 3 & 1 & 0 \\
\hline Valor medio (\%) & 15,70 & 16,48 & 2,31 & 2,35 & 2,90 & 2,96 & 25,10 & 25,26 \\
\hline \multicolumn{9}{|l|}{ Repetibilidad } \\
\hline$S_{r}$ & 0,73 & 0,79 & 0,29 & 0,24 & 0,31 & 0,16 & 1,33 & 0,90 \\
\hline$r$ & 2,03 & 2,20 & 0,82 & 0,67 & 0,86 & 0,44 & 3,72 & 2,5 \\
\hline$R D S_{r}(\%)$ & 4,6 & 4,8 & 12,6 & 10,2 & 10,5 & 5,3 & 5,3 & 3,6 \\
\hline \multicolumn{9}{|l|}{ Reproducibilidad } \\
\hline$S_{R}$ & 1,88 & 1,54 & 0,44 & 0,33 & 0,42 & 0,49 & 1,99 & 1,90 \\
\hline$R$ & 5,27 & 4,32 & 1,22 & 0,91 & 1,18 & 1,36 & 5,58 & 5,33 \\
\hline$R S D_{R}(\%)$ & 12,0 & 9,4 & 18,8 & 13,8 & 14,6 & 16,4 & 7,9 & 7,5 \\
\hline $\mathrm{Ho}_{\mathrm{R}}$ & 1,1 & 0,9 & 1,3 & 1,0 & 1,1 & 1,2 & 0,8 & 0,8 \\
\hline
\end{tabular}

a Cromatografía de Capa Fina de Esteroles

b Cromatografía Líquida de Alta Eficacia de Esteroles y Dialcoholes

\section{CONCLUSIONES}

Los resultados obtenidos en la determinación por el método CCF-esteroles (oficial) muestran una mejora de precisión con respecto a ensayos colaborativos anteriores, como consecuencia de la experiencia adquirida por los laboratorios en la aplicación del método. La reproducibilidad es aceptable en las muestras de aceite de oliva pero es baja en las de aceite de orujo.

El método de análisis CLAE-esteroles + dialcoholes muestra una precisión aceptable en la determinación del $\Delta 7$-estigmastenol, tanto en los aceites de oliva como de orujo. Esto se atribuye a que se elimina la posibilidad de interferencia positiva, probablemente debida al cicloartenol o a compuestos que en CCF eluyen con el eritrodiol, y a que no tiene lugar la ambigüedad del raspado la banda de esteroles que es consustancial con el método CCF-esteroles. Por otra parte se observa un aumento de los valores de los $\Delta 7$-esteroles, con respecto al método oficial.

La adopción del método CLAE-esteroles + dialcoholes por los laboratorios de las industrias oleícolas reportaría un ahorro económico y de tiempo al permitir el análisis de esteroles y eritrodiol + uvaol en una sola determinación. Además la mayor precisión del método daría mayor confianza en el resultado obtenido. Finalmente, el incremento del valor del $\Delta 7$-estigmastenol obtenido por este método reduciría la probabilidad de que el análisis por el método oficial diese valores más altos, suceso posible dada la reproducibilidad que muestra el método oficial.

La adopción de este método en los reglamentos que regulan el comercio del aceite de oliva, disminuiría los casos de discrepancia de resultados entre laboratorios, dada su mayor precisión. Sin embargo habría que efectuar una revisión del límite máximo permitido para el $\Delta 7$ estigmastenol, ya que el actual está establecido con los datos obtenidos mediante determinaciones con el método CCF-esteroles, que para los $\Delta 7$-esteroles origina valores significativamente inferiores. Ello implicaría la obtención de un banco de datos por aplicación del nuevo método a aceites de oliva y de orujo genuinos con alto contenido en $\Delta 7$-esteroles.

\section{AGRADECIMIENTOS}

Los autores desean expresar su agradecimiento a los responsables de los laboratorios participantes en el estudio colaborativo: Aceites del Sur, S. A. (Sevilla); Aceites Toledo, S. A. (Los Yébenes); Agra, S. A. (Gandía); ANIERAC (Madrid); Coreysa (Osuna); Gaspar Peral y Cía (Valencia); Instituto de la Grasa (Sevilla); Juan Ballester Rosés (Tortosa); Koipe (Andújar); Laboratori Agrari de la Generalitat de Calalunya (Cabrils); Laboratorio Agroalimentario de Granada (Atarfe); Laboratorio Agroalimentario de Madrid, Laboratorio Arbitral del M.A.P.A. (Madrid); Laboratorio de 
Veterinaria Militar (Madrid); Laboratorio Municipal de Barcelona, Laboratorio Municipal de Higiene (Madrid); Minerva, S. A. (Málaga) y SENPA (Madrid).

Asimismo agradecen la colaboración de D. Manuel Rodríguez Aguilar por la preparación de las muestras y a la CICYT por el soporte económico (Proyecto ALI940782).

\section{BIBLIOGRAFÍA}

Amelio, M., Rizzo, R. y Varazini, F. (1992). -«Determination of sterols, erythrodiol, uvaol and alkanols in olive oil, using combined solid-phase extraction, high-performance liquid chromatographic and high-resolution gas chromatographic techniques".- J. Chromatogr. 606, 179185.

Biedermann, M., Grob, K. y Mariani, C. (1989). - Transesterification and on-line LC-GC for determining the sum of free and esterified sterols in edible oils and fats.- Fat Sci. Technol. 95, 127-133.

Horwitz, H. (1988). - «Protocol for the design, conduct and interpretation of collaborative studies".- Pure Appl. Chem. 60, 855-864.
Lanuzza, F., Micali, G. y Calabrò, G. (1995). - «Sterol analysis in olive oil by transesterification and HPLC-HRGC coupling".- Riv. Ital Sostanze Grasse 72, 105-109.

León, M. y Cert, A. (1994). - «Recomendaciones para la aplicación de algunos métodos analíticos incluidos en el Reglamento CEE 2568/91 relativo a las características de los aceites de oliva y de orujo de oliva»-Grasas y Aceites 45, 395-401.

Morchio, G. et al. (1989). - «Análisis cromatográfico de esteroles. Resultados de un estudio colaborativo".- Riv. Ital. Sostanze Grasse 66, 531-538.

Moreda, W., Pérez-Camino, M. C. y Cert, A. (1995). - «Determinación de algunos parámetros de pureza en aceites de oliva. Resultados de un estudio colaborativo".- Grasas y Aceites 46, 279-284.

Pocklington, W. D. (1991). - «Precision and accuracy of analysis, standardisation of analytical methods" en "Analysis of Oilseeds, Fats and Fattyfoods", J. B. Rossell and J. L. Pritchard (Eds.).- Elsevier Applied Science Publishers, p. 1.

Reglamento CEE 2568/91 de la Comisión (1991). -«Relativo a las características de los aceites de oliva y de orujo de oliva y sobre sus métodos de análisis. Anexos $\mathrm{V}$ y VI’.Diario Oficial L248, 15-24.

Recibido: Marzo 1997 Aceptado: Julio 1997 